\title{
Global existence and exponential decay of the solution for a viscoelastic wave equation with a delay
}

\author{
Qiuyi Dai ${ }^{\dagger} \quad$ Zhifeng Yang $^{\dagger} \ddagger *$ \\ $\dagger$ College of Mathematics and Computer Science, Hunan Normal University \\ Changsha, Hunan, 410081, P.R.China \\ $\ddagger$ Department of Mathematics and Computational Science, Hengyang Normal University \\ Hengyang, Hunan, 421008, P.R.China
}

\begin{abstract}
In this paper, we consider initial-boundary value problem of viscoelastic wave equation with a delay term in the interior feedback. Namely, we study the following equation

$$
u_{t t}(x, t)-\Delta u(x, t)+\int_{0}^{t} g(t-s) \Delta u(x, s) d s+\mu_{1} u_{t}(x, t)+\mu_{2} u_{t}(x, t-\tau)=0
$$

together with initial-boundary conditions of Dirichlet type in $\Omega \times(0,+\infty)$, and prove that for arbitrary real numbers $\mu_{1}$ and $\mu_{2}$, the above mentioned problem has a unique global solution under suitable assumptions on the kernel $g$. This improve the results of the previous literature such as [6] and [13] by removing the restriction imposed on $\mu_{1}$ and $\mu_{2}$. Furthermore, we also get an exponential decay results for the energy of the concerned problem in the case $\mu_{1}=0$ which solves an open problem proposed by M. Kirane and B. Said-Houari in [13].
\end{abstract}

Keywords viscoelastic wave equation; global existence; energy decay; interior feedback.

Mathematics Subject Classification (2010) 35L05, 35L20, 35L70, 93D15

\section{Introduction}

It is well-known that the free vibration of membrane can be described by wave equation of the form

$$
u_{t t}(x, t)-\Delta u(x, t)=0
$$

in $\Omega \times(0,+\infty)$, subjected to initial conditions and some boundary conditions. Here, $\Omega \subset \mathbf{R}^{2}$ is a bounded domain. For the case of high dimension, there are also many discussions in mathematical physics. For the better comprehension of our motivation, we appeal to readers to keep in mind that the system (1.1) is conservative.

\footnotetext{
${ }^{*}$ Corresponding author. E-mail: zhifeng _ yang@126.com.
} 
To study the propagation mechanism of wave, two main factors, that is damping and timedelay, are taken into consideration. When the damping occurs, the corresponding mathematical model becomes

$$
u_{t t}(x, t)-\Delta u(x, t)+\mu u_{t}=0,
$$

and the energy of this system is dissipative. We here point out that the results about the models (1.1) and (1.2) are quite abundant. And what is regretful is that we cannot list these literatures one by one.

Moreover, time-delay effects often appears in many daily life practical problems. These hereditary effects are sometime unavoidable, and it may turn a well-behaved system into a wild one. That is to say, they can induce some instabilities(see for instance[1-5] and the references therein). The stability issue of systems with delay is, therefore, of theoretical and practical importance.

For the wave equation with boundary or interior delay, S. Nicaise, C. Pignotti, M. Gugat and G. Leugering et al. obtained some profound results[6-8,20-22]. In [6], the authors examined a system of wave equation with the damping and the time-delay inside the domain. Namely, they considered the following system

$$
\begin{cases}u_{t t}(x, t)-\Delta u(x, t)+\mu_{1} u_{t}(x, t)+\mu_{2} u_{t}(x, t-\tau)=0, & x \in \Omega, t>0, \\ u(x, t)=0, & x \in \partial \Omega, t \geq 0, \\ u(x, 0)=u_{0}(x), \quad u_{t}(x, 0)=u_{1}(x), & x \in \Omega, \\ u_{t}(x, t-\tau)=f_{0}(x, t-\tau), & x \in \Omega, 0<t<\tau,\end{cases}
$$

and proved that the energy of the above problem is exponentially decay (or the trivial solution is exponentially stable) provided that $0<\mu_{2}<\mu_{1}$. Furthermore, it is also showed in the case $\mu_{2} \geq \mu_{1}$ that there exists a sequence of arbitrary small (or large) delays such that instabilities occur. The same results were showed when both the damping and the delay act on the boundary. Particularly, if $\mu_{1}=0$, that is, if we have only the delay part in the boundary condition, the system becomes unstable (see [2]). From these results, we may figure out that the delay term lead to instability of the system. And to avoid this problem, M. Gugat considered feedback laws where a certain delay is included as a part of the control law and not as a perturbation, and obtained the exponential stability of the proposed feedback with retarded input (see [20]). In addition, M. Gugat and G. Leugering considered the feedback stabilization of quasilinear hyperbolic systems on star-shaped networks. They obtained the exponential stability of the system by introducing an $L^{2}$-Lyapunov function with delay terms (see [21]). Recently, C. Pignotti considered the wave equation with internal distributed time delay and local damping in a bounded and smooth domain, and showed that an exponential stability result holds if the coefficient of the delay term is sufficiently small(see [22]).

In this paper, we are concerned with the initial-boundary value problem(IBVP) of the vis- 
coelastic wave equation

$$
\left\{\begin{array}{rlrl}
u_{t t}(x, t)-\Delta u(x, t)+\int_{0}^{t} g(t-s) \Delta u(x, s) d s & \\
& +\mu_{1} u_{t}(x, t)+\mu_{2} u_{t}(x, t-\tau)=0, & & x \in \Omega, t>0, \\
u(x, t)=0, & & x \in \partial \Omega, t \geq 0, \\
u(x, 0)=u_{0}(x), \quad u_{t}(x, 0)=u_{1}(x), & & x \in \Omega, \\
u_{t}(x, t-\tau)=f_{0}(x, t-\tau), & & x \in \Omega, 0<t<\tau,
\end{array}\right.
$$

where $\Omega \subset \mathbf{R}^{n}, n \geq 1$ be a regular and bounded domain with a boundary $\partial \Omega$ of class $C^{2}$. Moreover, $\mu_{1}, \mu_{2}$ are two real numbers, $\tau>0$ represents the time delay and $u_{0}, u_{1}, f_{0}$ are given initial data belonging to suitable spaces.

System (1.5) comes from linear models for propagation of viscoelastic wave in a compressible fluid. In mechanics, it is well known that solid and fluid materials exhibit not only elasticity but also hereditary properties. The hereditary properties are described by viscoelasticity, where the mechanical response of the materials is taken to be influenced by the previous behavior of the materials themselves. In other words, the viscoelastic materials exhibit memory effects. This leads to a constitutive relationship between the stress and strain involving the convolution of the strain with a relaxation function. This term is called a "memory term". From the mathematical point of view, these hereditary properties are modeled by integro-differential operators.

To motivate our present work, let us recall some previous results regarding the viscoelastic wave equation. In the absence of time-delay, the viscoelastic wave equation of the form

$$
u_{t t}-\Delta u+\int_{0}^{t} g(t-s) \Delta u(x, s) d s+a(x) u_{t}=0
$$

in $\Omega \times(0, \infty)$, subjected to initial conditions and boundary conditions of Dirichlet type has been considered by Cavalcanti et al.[9]. They showed an exponential decay result under some restrictions on $a(x)$ and $g(t)$. To be specific, they assumed that $a: \Omega \rightarrow \mathbf{R}$ is a nonnegative and bounded function, and the kernel $g$ in the memory term decays exponentially. This result has been improved later by Berrimi and Messaoudi[10] under weaker conditions on both $a$ and $g$. And then, more general problems than the one considered in [9] are studied in [11,12]. Some profound results about the relationship between the stability of solutions and the relaxation kernel $g$ are obtained. It is worthwhile to note that, if $a(x) \equiv 0$ in (1.6), some results about the energy decay are obtained by many researchers. See, for instance Tatar[18], Mustafa and Messaoudi[19].

Then a nature problem is that what would happen when a delay term occurs in (1.6). In this case, M. Kirane and B. Said-Houari studied problem (1.5) in [13] with coefficients $\mu_{1}$ and $\mu_{2}$ positive. They showed that problem (1.5) has a unique weak solution (see [13], Theorem 3.1 ), and its energy is exponentially decay provided that $\mu_{2} \leq \mu_{1}$. It is worth pointing out that the assumptions $\mu_{1}>0$ and $\mu_{2} \leq \mu_{1}$ play a decisive role in proof of the above mentioned results. Because the authors must use the damping term $\mu_{1} u_{t}(x, t)$ to control the delay term 
$\mu_{2} u_{t}(x, t-\tau)$ in the priori estimate of the solution and the decay estimate of the energy. This makes the authors arise an open problem that whether the decay property of the energy they have obtained are preserved in the case $\mu_{1}=0$ ? By the way, the results of [13] has been generalized recently by W.J. Liu to a system with time dependent delay in [14]. The method used by W.J. Liu is very similar to those used in [13].

The aims of the present paper are twofold. At first, we aim to prove an existence result of problem (1.5) without restrictions of $\mu_{1}, \mu_{2}>0$ and $\mu_{2} \leq \mu_{1}$. Unlike in [13], our new idea is to control the delay term by the derivative of the energy instead of by the damping term in the priori estimate of solutions. In the second, we will give a positive answer to the open problem proposed by M. Kirane and B. Said-Houari in [13]. That is we will prove a energy decay result for problem (1.5) in the case $\mu_{1}=0$. The main difficulty in handling this problem is that we have no damping term to control the delay term in the estimate of the energy decay. To overcome this difficulty, our basic idea is to control the delay term by making use of the viscoelasticity term. And in order to achieve this goal, a restriction of the size between the parameter $\mu_{2}$ and the kernel $g$ and a new Lyapunov functional, different from that one used in [13], are needed.

The paper is organized as follows. In Section 2 we give some preliminaries. In Section 3, we prove the existence and the uniqueness of the solution of problem $(1,5)$. In Section 4, we prove the exponential decay of the energy of this problem in the case $\mu_{1}=0$.

\section{Preliminaries}

In this section, we shall prepare some materials needed in the proof of our result and state. We use the standard Lebesgue space $L^{2}(\Omega)$ and Sobolev space $H_{0}^{1}(\Omega)$ with their usual norms $\|\cdot\|_{2}$ and $\|\cdot\|_{H_{0}^{1}}$ respectively. And we will write $(\cdot, \cdot)$ to denote the inner product in $L^{2}(\Omega)$. Throughout this paper, $C$ and $C_{i}$ are used to denote generic positive constants.

We first state the general assumptions on the kernel function $g$ as follows:

(A1) $g: \mathbf{R}^{+} \rightarrow \mathbf{R}^{+}$is a $C^{1}$ function satisfying

$$
g(0)>0, \quad 1-\int_{0}^{\infty} g(s) d s=l>0 .
$$

(A2) There exists a positive constant $\zeta$ such that

$$
g^{\prime}(t) \leq-\zeta g(t), \quad \forall t>0
$$

A typical example of such function is

$$
g(t)=e^{-a t}, a>1 .
$$

Now,let us introduce the following notation:

$$
(\phi \circ \psi)(t):=\int_{0}^{t} \phi(t-s) \int_{\Omega}|\psi(t)-\psi(s)|^{2} d x d s .
$$


For any function $\phi \in C^{1}(\mathbf{R})$ and any $\psi \in H^{1}\left(0, T, L^{2}(\Omega)\right)$, straightforward computations lead to(see $[13,15])$

$$
\begin{aligned}
& \int_{\Omega} \psi_{t}(t) \int_{0}^{t} \phi(t-s) \psi(s) d s d x \\
& \quad=\frac{1}{2}\left(\phi^{\prime} \circ \psi\right)(t)-\frac{1}{2} \phi(t)\|\psi\|_{2}^{2}-\frac{1}{2} \frac{d}{d t}\left\{(\phi \circ \psi)(t)-\left(\int_{0}^{t} \phi(s) d s\right)\|\psi\|_{2}^{2}\right\} .
\end{aligned}
$$

The following lemma will be used later in order to define the new modified energy functional of problem (1.5).

Lemma 2.1(see [13, Lemma 2.2] or [16]). For $u \in L^{2}\left(0, T, H_{0}^{1}(\Omega)\right)$, we have

$$
\int_{\Omega}\left(\int_{0}^{t} g(t-s)(u(t)-u(s)) d s\right)^{2} d x \leq(1-l) C_{*}^{2}(g \circ \nabla u)(t),
$$

where $C_{*}$ is the Poincaré constant and $l$ is given in (A1).

Let

$$
U=\left\{u \mid u \in L^{2}\left(0, T ; H_{0}^{1}(\Omega)\right), \quad u_{t} \in L^{2}\left(0, T ; L^{2}(\Omega)\right)\right\}
$$

and

$$
V=\left\{v \mid v \in U, \quad v(x, T)=0, \quad v_{t}(x, T)=0\right\} .
$$

Next, we give the definition of the weak solution of problem (1.5).

Definition 2.2 We say a function $u \in U$ is a weak solution of the initial-boundary value problem (1.5) provided

$$
\begin{array}{r}
-\int_{0}^{T}\left(u_{t}, v_{t}\right) d t+\int_{0}^{T}(\nabla u(t), \nabla v(t)) d t-\int_{0}^{T} \int_{0}^{t} g(t-s)(\nabla u(s), \nabla v(s)) d s d t \\
+\mu_{1} \int_{0}^{T}\left(u_{t}(t), v\right) d t+\mu_{2} \int_{0}^{T}\left(u_{t}(t-\tau), v\right) d t+\left(v(x, 0), u_{1}(x)\right)=0
\end{array}
$$

for each $v \in V$ and

$$
u(x, 0)=u_{0}, \quad u_{t}(x, 0)=u_{1}, \quad u_{t}(x, t-\tau)=f_{0}(x, t-\tau), \quad t \in(0, \tau) .
$$

Remark 2.3 In view of Theorem 2 in $§ 5.92$ in [23], we know $u \in C\left(0, T ; L^{2}(\Omega)\right)$ and $u_{t} \in C\left(0, T ; H^{-1}(\Omega)\right)$. Consequently the equalities (2.5) above make sense.

\section{Existence of weak solutions}

In this section, we will give a sufficient condition that guarantees the well-posedness of the problem (1.5). We will use the Faedo-Galerkin approximation to prove it. So we will need the following prior estimates.

Theorem 3.1(Prior estimates) For all $u \in U$, there exists a constant $C$, depending only on $\Omega$ and $T$, such that

$$
\mathcal{E}(u(t)) \leq C,
$$


where

$$
\mathcal{E}(u(t))=\frac{1}{2}\left(\left\|u_{t}\right\|_{2}^{2}+\left(1-\int_{0}^{t} g(s) d s\right)\|\nabla u\|_{2}^{2}+(g \circ \nabla u)(t)\right) .
$$

Proof Since functions of the space $C^{\infty}((0, T) \times \Omega)$ are dense in the space $U$, we just need to prove the conclusion in the case of $u \in C^{\infty}((0, T) \times \Omega)$. So, the following calculations are all in this space.

Multiplying the first equation in (1.5) by $u_{t}$ and integrating on $\Omega$, straightforward computations lead to

$$
\mathcal{E}^{\prime}(u(t))=\frac{1}{2}\left(\left(g^{\prime} \circ \nabla u\right)(t)-g(t)\|\nabla u(t)\|_{2}^{2}\right)-\mu_{1} \int_{\Omega} u_{t}^{2}(t) d x-\mu_{2} \int_{\Omega} u_{t}(t-\tau) u_{t}(t) d x .
$$

By the assumptions (A1) and (A2) about $g(t)$, we have

$$
\left(g^{\prime} \circ \nabla u\right)(t)-g(t)\|\nabla u(t)\|_{2}^{2}<0 .
$$

So, thanks to the Cauchy-Schwartz inequality, we deduce

$$
\mathcal{E}^{\prime}(u(t)) \leq \frac{\left|\mu_{2}\right|}{2} \int_{\Omega} u_{t}^{2}(t-\tau) d x+\left(\frac{\left|\mu_{2}\right|}{2}+\left|\mu_{1}\right|\right) \int_{\Omega} u_{t}^{2}(t) d x
$$

By integrating on $[0, t]$, we have

$$
\mathcal{E}(u(t)) \leq \frac{\left|\mu_{2}\right|}{2} \int_{0}^{t} \int_{\Omega} u_{s}^{2}(s-\tau) d x d s+\left(\frac{\left|\mu_{2}\right|}{2}+\left|\mu_{1}\right|\right) \int_{0}^{t} \int_{\Omega} u_{s}^{2}(s) d x d s+\mathcal{E}(u(0)),
$$

where $\mathcal{E}(u(0))$ is a nonnegative constant.

Now, using the past history values about $u_{t}(t), \quad t \in[-\tau, 0]$, the first term in the right-hand side of (3.5) can be rewritten as follows

$$
\begin{aligned}
& \int_{0}^{t} \int_{\Omega} u_{s}^{2}(s-\tau) d x d s \\
& =\int_{\Omega} \int_{-\tau}^{t-\tau} u_{\rho}^{2}(\rho) d \rho d x \\
& =\int_{\Omega} \int_{-\tau}^{0} u_{\rho}^{2}(\rho) d \rho d x+\int_{\Omega} \int_{0}^{t-\tau} u_{\rho}^{2}(\rho) d \rho d x \\
& =\int_{\Omega} \int_{-\tau}^{0} f_{0}^{2}(\rho) d \rho d x+\int_{\Omega} \int_{0}^{t-\tau} u_{\rho}^{2}(\rho) d \rho d x \\
& \leq \int_{\Omega} \int_{-\tau}^{0} f_{0}^{2}(\rho) d \rho d x+\int_{\Omega} \int_{0}^{t} u_{\rho}^{2}(\rho) d \rho d x
\end{aligned}
$$

From (3.5) and (3.6), we obtain

$$
\mathcal{E}(u(t)) \leq\left(\left|\mu_{2}\right|+\left|\mu_{1}\right|\right) \int_{\Omega} \int_{0}^{t} u_{\rho}^{2}(\rho) d \rho d x+\frac{\left|\mu_{2}\right|}{2} \int_{\Omega} \int_{-\tau}^{0} f_{0}^{2}(\rho) d \rho d x+\mathcal{E}(u(0)) .
$$

Thus, we have

$$
\mathcal{E}(u(t)) \leq 2\left(\left|\mu_{2}\right|+\left|\mu_{1}\right|\right) \int_{0}^{t} \mathcal{E}(u(s)) d s+\frac{\left|\mu_{2}\right|}{2} \int_{\Omega} \int_{-\tau}^{0} f_{0}^{2}(\rho) d \rho d x+\mathcal{E}(u(0)) .
$$


By the Gronwall inequality, once $T>0$ be given, $\forall t \in[0, T]$, we have

$$
\mathcal{E}(u(t)) \leq\left(\frac{\left|\mu_{2}\right|}{2} \int_{\Omega} \int_{-\tau}^{0} f_{0}^{2}(\rho) d \rho d x+\mathcal{E}(u(0))\right) e^{2\left(\left|\mu_{2}\right|+\left|\mu_{1}\right|\right) T}, \quad \forall t \in[0, T] .
$$

Denote

$$
C=\left(\frac{\left|\mu_{2}\right|}{2} \int_{\Omega} \int_{-\tau}^{0} f_{0}^{2}(\rho) d \rho d x+\mathcal{E}(u(0))\right) e^{2\left(\left|\mu_{2}\right|+\left|\mu_{1}\right|\right) T} .
$$

Then we have $\mathcal{E}(u(t)) \leq C$.

With the above prior estimates, it is easy to prove the uniqueness of solutions as follows:

Theorem 3.2(uniqueness of solution). There exists at most one solution of problem (1.5) in the sense of definition 2.2.

Proof It suffices to show that the only weak solution of (1.5) with $u_{0}=u_{1}=f_{0}=0$ is

$$
u \equiv 0 \text {. }
$$

According to the energy estimate (3.9) in Theorem 3.1, and noting that $f_{0}=0, \mathcal{E}(u(0))=0$, we obtain

$$
\mathcal{E}(u(t))=0, \quad \forall t \in[0, T]
$$

So, we have

$$
\left\|u_{t}(t)\right\|_{2}=\|\nabla u(t)\|_{2}=0, \quad \forall t \in[0, T] .
$$

And this implies (3.10). Thus, we conclude that problem (1.5) has at most one solution.

Next, we give the existence of weak solution by the following theorem.

Theorem 3.3(Existence of weak solution). Assume that (A1) and (A2) hold. Then given $u_{0} \in H_{0}^{1}(\Omega), u_{1} \in L^{2}(\Omega), f_{0} \in L^{2}(-\tau, 0 ; \Omega)$ and $T>0$, there exists a weak solution $u$, defined by definition 2.2, of problem (1.5) on $(0, T)$.

Proof We first construct the approximate solution of (1.5) by Galerkin's method. Let $\left\{\lambda_{j}\right\}_{j=1}^{\infty}$ be the eigenvalue of the following eigenvalue problem:

$$
\begin{cases}-\Delta w=\lambda w, & x \in \Omega, \\ w=0, & x \in \partial \Omega .\end{cases}
$$

and $w_{j}$ is the eigenfunctions associated with $\lambda_{j}(j=1,2, \cdots)$. Furthermore, we choose $\left\{w_{j}\right\}_{j=1}^{\infty}$ such that

$$
\int_{\Omega} w_{j} w_{k} d x= \begin{cases}1, & j=k \\ 0, & j \neq k\end{cases}
$$

So, we have

$$
\int_{\Omega} \nabla w_{j} \nabla w_{k} d x= \begin{cases}\lambda_{j}, & j=k, \\ 0, & j \neq k .\end{cases}
$$

Moreover, $\left\{w_{j}\right\}_{j=1}^{\infty}$ is the orthogonal bases in $L^{2}(\Omega)$ and in $H_{0}^{1}(\Omega)$. 
We will seek an approximate solution in the form

$$
u_{n}(t)=\sum_{j=1}^{n} \gamma_{n}^{j}(t) w_{j}
$$

where we intend to select the coefficients $\gamma_{n}^{j}(t)(0 \leq t \leq T, j=1, \cdots, n)$ to satisfy

$$
\begin{gathered}
\gamma_{n}^{j}(0)=\left(u_{0}, w_{j}\right), \quad \gamma_{n t}^{j}(0)=\left(u_{1}, w_{j}\right), \\
\gamma_{n t}^{j}(x, t-\tau)=f_{0}(x, t-\tau), \quad t \in(0, \tau),
\end{gathered}
$$

and

$$
\left(u_{n t t}, w_{j}\right)+\left(\nabla u_{n}(t), \nabla w_{j}(t)\right)-\int_{0}^{t} g(t-s)\left(\nabla u_{n}(s), \nabla w_{j}\right) d s+\mu_{1}\left(u_{n t}(t), w_{j}\right)+\mu_{2}\left(u_{n t}(t-\tau), w_{j}\right)=0 .
$$

By (3.16), (3.19) becomes the linear system of ODE

$$
\ddot{\gamma}_{n}^{j}(t)+\mu_{1} \dot{\gamma}_{n}^{j}(t)+\mu_{2} \dot{\gamma}_{n}^{j}(t-\tau)+\lambda_{j}\left(1-\int_{0}^{t} g(s) d s\right) \gamma_{n}^{j}=0,
$$

subject to the initial conditions (3.17) and the history value (3.18).

For the initial value problem (3.20), (3.17) and (3.18), we can use the contraction mapping principle to prove that there exists a unique solution $\gamma_{n}(t)=\left(\gamma_{n}^{1}(t), \cdots, \gamma_{n}^{n}(t)\right) \in C^{2}$ when $t$ is small enough. And then, by the above prior estimates(see Theorem 3.1), we have $\left\|\gamma_{n}\right\|_{H_{0}^{1}} \leq C$. So, we can extend this solution to the whole interval $[0, T]$.

Next, according to the prior estimates, we see that the sequence $\left\{u_{n}\right\}_{n=1}^{\infty}$ is bounded in $L^{2}\left(0, T ; H_{0}^{1}(\Omega)\right),\left\{u_{n t}\right\}_{n=1}^{\infty}$ is bounded in $L^{2}\left(0, T ; L^{2}(\Omega)\right)$.

As a consequence there exists a subsequence $\left\{u_{k}\right\}_{k=1}^{\infty} \subset\left\{u_{n}\right\}_{n=1}^{\infty}$ and $u \in L^{2}\left(0, T ; H_{0}^{1}(\Omega)\right)$, with $u_{t} \in L^{2}\left(0, T ; L^{2}(\Omega)\right)$ such that

$$
\begin{aligned}
& u_{k} \rightarrow u \text { weakly in } L^{2}\left(0, T ; H_{0}^{1}(\Omega)\right) \text {, as } k \rightarrow \infty, \\
& u_{k t} \rightarrow u_{t} \text { weakly in } L^{2}\left(0, T ; L^{2}(\Omega)\right) \text {, as } k \rightarrow \infty .
\end{aligned}
$$

Next, arguing as in [17, Theorem 3.1], we will prove that $u$ is the weak solution of problem (1.5). For this, we choose a function $v \in\left\{\eta \mid \eta \in C^{1}\left(0, T ; H_{0}^{1}(\Omega)\right), \eta(x, T)=0, \eta_{t}(x, T)=0\right\}$ of the form

$$
v(t)=\sum_{j=1}^{N} \gamma^{j}(t) w_{j},
$$

where $\left\{\gamma^{j}\right\}_{j=1}^{N}$ are smooth functions, and $N$ is a fixed integer.

We select $k \geq N$, multiply the equation (3.19) by $\gamma^{j}(t)$, sum $j=1, \cdots, N$, and then integrate with respect to $t$, to discover

$$
I+I I=0
$$


where

$$
I=\int_{0}^{T}\left[-\left(u_{k t}, v_{t}\right)+\left(\nabla u_{k}(t), \nabla v(t)\right)-\int_{0}^{t} g(t-s)\left(\nabla u_{k}(s), \nabla v(s)\right) d s\right] d t
$$

and

$$
I I=\int_{0}^{T}\left[\mu_{1}\left(u_{k t}(t), v\right)+\mu_{2}\left(u_{k t}(t-\tau), v\right)\right] d t+\left(v(x, 0), u_{k t}(x, 0)\right) .
$$

By $(3.21),(3.22)$ and passing to the limit in (3.24) as $k \rightarrow \infty$, we obtain

$$
\begin{aligned}
\int_{0}^{T}[- & \left.\left(u_{t}, v_{t}\right)+(\nabla u(t), \nabla v(t))-\int_{0}^{t} g(t-s)(\nabla u(s), \nabla v(s)) d s\right] d t \\
& +\int_{0}^{T}\left[\mu_{1}\left(u_{t}(t), v\right)+\mu_{2}\left(u_{t}(t-\tau), v\right)\right] d t+\left(v(x, 0), u_{1}(x)\right)=0
\end{aligned}
$$

for all functions $v \in V$, since functions of the form (3.23) are dense in this space. And thus, (2.4) holds.

We must now verify that the limit function $u$ satisfies the initial conditions and the history value, i.e.

$$
\begin{gathered}
u(0)=u_{0}, \quad u_{t}(0)=u_{1}, \\
u_{t}(x, t-\tau)=f_{0}(x, t-\tau), \quad t \in(0, \tau) .
\end{gathered}
$$

For this, choose any function $v \in C^{2}\left(0, T ; H_{0}^{1}(\Omega)\right)$, with $v(T)=v_{t}(T)=0$. Then integrating by parts with respect to $t$ in $(3.25)$, we find

$$
\begin{aligned}
\int_{0}^{T} & {\left[\left(v_{t t}, u\right)+(\nabla u(t), \nabla v(t))-\int_{0}^{t} g(t-s)(\nabla u(s), \nabla v(s)) d s\right] d t } \\
& +\int_{0}^{T}\left[\mu_{1}\left(u_{t}(t), v\right)+\mu_{2}\left(u_{t}(t-\tau), v\right)\right] d t=\left(u(0), v_{t}(0)\right)-\left(u_{t}(0), v(0)\right) .
\end{aligned}
$$

Similarly from (3.24) we deduce

$$
\begin{aligned}
\int_{0}^{T} & {\left[\left(v_{t t}, u_{k}\right)+\left(\nabla u_{k}(t), \nabla v(t)\right)-\int_{0}^{t} g(t-s)\left(\nabla u_{k}(s), \nabla v(s)\right) d s\right] d t } \\
& \quad+\int_{0}^{T}\left[\mu_{1}\left(u_{k t}(t), v\right)+\mu_{2}\left(u_{k t}(t-\tau), v\right)\right] d t=\left(u_{k}(0), v_{t}(0)\right)-\left(u_{k t}(0), v(0)\right) .
\end{aligned}
$$

Recalling (3.17),(3.21) and (3.22), we get

$$
\begin{aligned}
& \int_{0}^{T}\left[\left(v_{t t}, u\right)+(\nabla u(t), \nabla v(t))-\int_{0}^{t} g(t-s)(\nabla u(s), \nabla v(s)) d s\right] d t \\
& \quad+\int_{0}^{T}\left[\mu_{1}\left(u_{t}(t), v\right)+\mu_{2}\left(u_{t}(t-\tau), v\right)\right] d t=\left(u_{0}, v_{t}(0)\right)-\left(u_{1}, v(0)\right),
\end{aligned}
$$

as $k \rightarrow \infty$. Comparing identities (3.28) and (3.30), we conclude (3.26), since $v(0), v_{t}(0)$ are arbitrary. In addition, $\forall j, \int_{\Omega} u_{k t}(t-\tau) w_{j} d x \rightarrow \int_{\Omega} f_{0}(t-\tau) w_{j} d x$ weakly in $L^{2}\left(0, T ; L^{2}(\Omega)\right)$ as $k \rightarrow \infty$. So we easily deduce $u_{t}(x, t-\tau)=f_{0}(x, t-\tau)$. And then (3.27) holds. Hence $u$ is a weak solution of (1.5) in the sense of Definition 2.2. 


\section{Exponential energy decay in the case $\mu_{1}=0$}

In this section, we will give the exponential energy decay result about the problem (1.5) in the case $\mu_{1}=0$. That is to say, we will study the following system

$$
\left\{\begin{array}{l}
u_{t t}(x, t)-\Delta u(x, t)+\int_{0}^{t} g(t-s) \Delta u(x, s) d s+\mu u_{t}(x, t-\tau)=0, \quad x \in \Omega, t>0 \\
u(x, t)=0, \quad x \in \partial \Omega, \quad t \geq 0 \\
u(x, 0)=u_{0}(x), \quad u_{t}(x, 0)=u_{1}(x), \quad x \in \Omega \\
u_{t}(x, t-\tau)=f_{0}(x, t-\tau), \quad x \in \Omega, 0<t<\tau
\end{array}\right.
$$

where $\mu$ is a real number.

We first define the classical energy by

$$
e(t)=\frac{1}{2}\left(\left\|u_{t}\right\|_{2}^{2}+\|\nabla u\|_{2}^{2}\right), \quad t \geq 0 .
$$

Then by the first equation in (4.1) it is easy to see that

$$
e^{\prime}(t)=\int_{\Omega} \nabla u_{t} \int_{0}^{t} g(t-s) \nabla u(s) d s d x-\mu \int_{\Omega} u_{t}(t) u_{t}(t-\tau) d x, \quad t \geq 0 .
$$

By (2.2) and inspired by [13], we define the new modified energy functional of problem (4.1) as follows:

$$
E(t)=\frac{1}{2}\left(\left\|u_{t}\right\|_{2}^{2}+\left(1-\int_{0}^{t} g(s) d s\right)\|\nabla u\|_{2}^{2}+(g \circ \nabla u)(t)\right)+\frac{\xi}{2} \int_{t-\tau}^{t} \int_{\Omega} e^{\sigma(s-t)} u_{t}^{2}(x, s) d x d s,
$$

where $\sigma$ and $\xi$ are two positive constants to be determined later.

Since the function $g$ is positive, continuous and $g(0)>0$, then for any $t \geq t_{0}>0$, we have

$$
\int_{0}^{t} g(s) d s \geq \int_{0}^{t_{0}} g(s) d s=g_{0} .
$$

Our stability result reads as follows:

Theorem 4.1(Exponential energy decay) Let $u$ be the solution of (4.1). Assume that $g$ satisfies (A1) and (A2), and $|\mu|<a$, where $a$ is a positive constant defined by (4.31). Then, for any $t_{0}>0$, there exists two positive constants, $K$ and $\lambda$, such that the energy of problem (4.1) satisfies

$$
E(t) \leq K e^{-\lambda \zeta\left(t-t_{0}\right)}, \quad \forall t \geq t_{0},
$$

where the function $\zeta$ satisfies the assumption (A2).

Proof. We define the Lyapunov functional

$$
L(t):=E(t)+\varepsilon_{1} \Psi(t)+\varepsilon_{2} \chi(t),
$$

where $\varepsilon_{i}, i=1,2$ are two positive real numbers which will be chosen later, and

$$
\Psi(t):=\int_{\Omega} u u_{t} d x
$$




$$
\chi(t):=-\int_{\Omega} u_{t} \int_{0}^{t} g(t-s)(u(t)-u(s)) d s d x .
$$

Remark 4.2 We deduce that, for all $\varepsilon_{i}>0, i=1,2$, the Lyapunov functional $L(t)$ and the energy $E(t)$ are equivalent in the sense that there exist two positive constants $\beta_{1}, \beta_{2}$ depending on $\varepsilon_{1}$ and $\varepsilon_{2}$ such that

$$
\beta_{1} E(t) \leq L(t) \leq \beta_{2} E(t), \quad \forall t \geq 0 .
$$

In fact, using Young's inequality, Poincaré's inequality and (2.3), we have

$$
\varepsilon_{2}|\chi(t)| \leq \frac{\varepsilon_{2}}{2}\left\|u_{t}\right\|_{2}^{2}+\frac{\varepsilon_{2}(1-l) C_{*}^{2}}{2}(g \circ \nabla u)(t)
$$

and

$$
\varepsilon_{1}|\Psi(t)| \leq \frac{\varepsilon_{1}}{2}\left\|u_{t}\right\|_{2}^{2}+\frac{\varepsilon_{1} C_{*}}{2}\|\nabla u\|_{2}^{2} .
$$

So, we obtain $|L(t)| \leq C E(t)$, where $C>0$ is a constant. And then (4.8) holds.

Now, we will estimate the derivative of $L(t)$ according to the following steps.

Step 1: Estimate of the derivative of $E(t)$.

Differentiating (4.2), we have

$$
\begin{aligned}
E^{\prime}(t)= & \int_{\Omega}\left(u_{t} u_{t t}+\left(1-\int_{0}^{t} g(s) d s\right) \nabla u \nabla u_{t}-\frac{1}{2} g(t)|\nabla u|^{2}\right) d x \\
& +\int_{0}^{t} g(t-s) \int_{\Omega} \nabla u_{t}(t)(\nabla u(t)-\nabla u(s)) d x d s \\
& +\frac{1}{2} \int_{0}^{t} g^{\prime}(t-s) \int_{\Omega}|\nabla u(t)-\nabla u(s)|^{2} d x d s \\
& +\frac{\xi}{2} \int_{\Omega} u_{t}^{2}(x, t) d x-\frac{\xi}{2} \int_{\Omega} e^{-\sigma \tau} u_{t}^{2}(x, t-\tau) d x \\
& -\frac{\sigma \xi}{2} \int_{t-\tau}^{t} \int_{\Omega} e^{-\sigma(t-s)} u_{t}^{2}(x, s) d x d s .
\end{aligned}
$$

Using the first equation of (4.1), we obtain

$$
\begin{aligned}
E^{\prime}(t) & =\frac{1}{2}\left(g^{\prime} \circ \nabla u\right)(t)-\frac{1}{2} g(t)\|\nabla u\|_{2}^{2}-\mu \int_{\Omega} u_{t}(t) u_{t}(t-\tau) d x \\
& +\frac{\xi}{2}\left\|u_{t}\right\|_{2}^{2}-\frac{\xi}{2} e^{-\sigma \tau} \int_{\Omega} u_{t}^{2}(x, t-\tau) d x-\frac{\sigma \xi}{2} \int_{t-\tau}^{t} \int_{\Omega} e^{-\sigma(t-s)} u_{t}^{2}(x, s) d x d s .
\end{aligned}
$$

And then, by Cauchy inequalities, we get

$$
\begin{gathered}
E^{\prime}(t) \leq \frac{1}{2}\left(g^{\prime} \circ \nabla u\right)(t)+\left(\frac{|\mu|}{2}+\frac{\xi}{2}\right)\left\|u_{t}\right\|_{2}^{2}+\left(\frac{|\mu|}{2}-\frac{\xi}{2} e^{-\sigma \tau}\right) \int_{\Omega} u_{t}^{2}(x, t-\tau) d x \\
-\frac{1}{2} g(t)\|\nabla u\|_{2}^{2}-\frac{\sigma \xi}{2} \int_{t-\tau}^{t} \int_{\Omega} e^{-\sigma(t-s)} u_{t}^{2}(x, s) d x d s .
\end{gathered}
$$

Step 2: Estimate of the derivative of $\Psi(t)$. 
Differentiating and integrating by parts, we obtain

$$
\begin{aligned}
\Psi^{\prime}(t)= & \left\|u_{t}\right\|_{2}^{2}+\int_{\Omega} u\left(\Delta u-\int_{0}^{t} g(t-s) \Delta u(s) d s-\mu u_{t}(t-\tau)\right) d x \\
= & \left\|u_{t}\right\|_{2}^{2}+\int_{\Omega} \nabla u \int_{0}^{t} g(t-s)(\nabla u(s)-\nabla u(t)) d s d x \\
& +\left(\int_{0}^{t} g(s) d s-1\right)\|\nabla u\|_{2}^{2}-\mu \int_{\Omega} u(t) u_{t}(t-\tau) d x \\
\leq & \int_{\Omega} \nabla u \int_{0}^{t} g(t-s)(\nabla u(s)-\nabla u(t)) d s d x \\
& +\left\|u_{t}\right\|_{2}^{2}-l\|\nabla u\|_{2}^{2}-\mu \int_{\Omega} u(t) u_{t}(t-\tau) d x .
\end{aligned}
$$

By Young's inequality and Lemma 2.1, we get(see [13])

$$
\begin{aligned}
& \int_{\Omega} \nabla u \int_{0}^{t} g(t-s)(\nabla u(s)-\nabla u(t)) d s d x \\
\leq & \delta_{1}\|\nabla u\|_{2}^{2}+\frac{1}{4 \delta_{1}} \int_{\Omega}\left(\int_{0}^{t} g(t-s)|\nabla u(s)-\nabla u(t)| d s\right)^{2} d x \\
\leq & \delta_{1}\|\nabla u\|_{2}^{2}+\frac{(1-l) C_{*}^{2}}{4 \delta_{1}}(g \circ \nabla u)(t) . \quad\left(\forall \delta_{1}>0\right)
\end{aligned}
$$

Also, using Young's and Poincaré's inequalities, we have

$$
-\mu \int_{\Omega} u(t) u_{t}(t-\tau) d x \leq \delta_{1}\|\nabla u\|_{2}^{2}+C\left(\delta_{1}\right) \int_{\Omega} u_{t}^{2}(t-\tau) d x .
$$

Combining (4.12)-(4.14) and choosing $\delta_{1}$ small enough, the estimate

$$
\Psi^{\prime}(t) \leq-\frac{l}{2}\|\nabla u\|_{2}^{2}+C_{1} \int_{\Omega}\left(u_{t}^{2}(t)+u_{t}^{2}(t-\tau)\right) d x+C_{2}(g \circ \nabla u)(t)
$$

holds for some positive constants $C_{i}, i=1,2$.

Step 3: Estimate of the derivative of $\chi(t)$.

Differentiating (4.7) and integrating by parts, we have

$$
\begin{aligned}
\chi^{\prime}(t)= & \left(1-\int_{0}^{t} g(s) d s\right) \int_{\Omega} \nabla u \int_{0}^{t} g(t-s)(\nabla u(t)-\nabla u(s)) d s d x \\
& +\int_{\Omega}\left(\int_{0}^{t} g(t-s)(\nabla u(s)-\nabla u(t)) d s\right)^{2} d x-\int_{\Omega} u_{t} \int_{0}^{t} g^{\prime}(t-s)(u(t)-u(s)) d s d x \\
& -\int_{0}^{t} g(s) d s\left\|u_{t}\right\|_{2}^{2}+\mu \int_{\Omega} u_{t}(t-\tau) \int_{0}^{t} g(t-s)(u(t)-u(s)) d s d x
\end{aligned}
$$

We first estimate the second item of (4.16) as follows:

$$
\int_{\Omega}\left(\int_{0}^{t} g(t-s)(\nabla u(s)-\nabla u(t)) d s\right)^{2} d x
$$




$$
\begin{aligned}
& =\int_{\Omega}\left(\int_{0}^{t} \sqrt{g(t-s)}(\sqrt{g(t-s)}(\nabla u(s)-\nabla u(t))) d s\right)^{2} d x \\
& \leq \int_{\Omega}\left(\int_{0}^{t} g(t-s)\right)\left(g(t-s)(\nabla u(s)-\nabla u(t))^{2} d s\right) d x \\
& \leq(1-l)(g \circ \nabla u)(t) .
\end{aligned}
$$

Then, using Young's inequality and Lemma 2.1, we get(see $[13,14])$

$$
\begin{gathered}
\left(1-\int_{0}^{t} g(s) d s\right) \int_{\Omega} \nabla u \int_{0}^{t} g(t-s)(\nabla u(t)-\nabla u(s)) d s d x \\
\leq \delta_{2}\|\nabla u\|_{2}^{2}+\frac{C_{3}}{\delta_{2}}(g \circ \nabla u)(t), \quad\left(\forall \delta_{2}>0\right) \\
-\int_{\Omega} u_{t} \int_{0}^{t} g^{\prime}(t-s)(u(t)-u(s)) d s d x \leq \delta_{2}\left\|u_{t}\right\|_{2}^{2}-\frac{C_{4}}{\delta_{2}}\left(g^{\prime} \circ \nabla u\right)(t)
\end{gathered}
$$

and

$$
\mu \int_{\Omega} u_{t}(t-\tau) \int_{0}^{t} g(t-s)(u(t)-u(s)) d s d x \leq \frac{C_{5}}{\delta_{2}}(g \circ \nabla u)(t)+\delta_{2} \int_{\Omega} u_{t}^{2}(t-\tau) d x
$$

where $C_{i}, i=3,4,5$ are some positive constants.

Combining (4.16)-(4.19) and (2.3), we obtain

$$
\begin{aligned}
\chi^{\prime}(t) \leq & \left(\delta_{2}-\int_{0}^{t} g(s) d s\right)\left\|u_{t}\right\|_{2}^{2}+\delta_{2}\|\nabla u\|_{2}^{2}+\frac{C_{6}}{\delta_{2}}(g \circ \nabla u)(t) \\
& -\frac{C_{7}}{\delta_{2}}\left(g^{\prime} \circ \nabla u\right)(t)+\delta_{2} \int_{\Omega} u_{t}^{2}(t-\tau) d x
\end{aligned}
$$

where $C_{i}, i=6,7$ are some positive constants.

Step 4: Estimate of the derivative of $L(t)$.

By using (4.3),(4.5),(4.11),(4.15) and (4.20), a series of computations yields, for $t \geq t_{0}$,

$$
\begin{aligned}
L^{\prime}(t) \leq & \left(\frac{|\mu|}{2}+\frac{\xi}{2}+\varepsilon_{1} C_{1}+\varepsilon_{2}\left(\delta_{2}-g_{0}\right)\right)\left\|u_{t}\right\|_{2}^{2}+\left(\varepsilon_{2} \delta_{2}-\frac{\varepsilon_{1} l}{2}\right)\|\nabla u\|_{2}^{2} \\
& +\left(\frac{1}{2}-\frac{\varepsilon_{2} C_{7}}{\delta_{2}}\right)\left(g^{\prime} \circ \nabla u\right)(t)+\left(\varepsilon_{1} C_{1}+\varepsilon_{2} \delta_{2}+\frac{|\mu|}{2}-\frac{\xi}{2 e^{\sigma \tau}}\right) \int_{\Omega} u_{t}^{2}(t-\tau) d x \\
& +\left(\varepsilon_{1} C_{2}+\frac{\varepsilon_{2} C_{6}}{\delta_{2}}\right)(g \circ \nabla u)(t)-\frac{\sigma \xi}{2} \int_{t-\tau}^{t} \int_{\Omega} e^{-\sigma(t-s)} u_{t}^{2}(s) d x d s .
\end{aligned}
$$

Now, we deduce that, for the positive constants $\xi, \delta_{2}, \varepsilon_{1}, \varepsilon_{2}$ and $\sigma$, the following system of inequalities

$$
\left\{\begin{array}{l}
\frac{|\mu|}{2}+\frac{\xi}{2}+\varepsilon_{1} C_{1}+\varepsilon_{2}\left(\delta_{2}-g_{0}\right)<0 \\
\varepsilon_{2} \delta_{2}-\frac{\varepsilon_{1} l}{2}<0 \\
\frac{1}{2}-\frac{\varepsilon_{2} C_{7}}{\delta_{2}}>0 \\
\varepsilon_{1} C_{1}+\varepsilon_{2} \delta_{2}+\frac{|\mu|}{2}-\frac{\xi}{2 e^{\sigma \tau}}<0,
\end{array}\right.
$$


is solvable only if we add some suitable conditions to $|\mu|$.

In fact, we can find solutions of (4.22) according to the following steps.

Step 1: We first pick $\delta_{2}$ small enough such that

$$
\delta_{2}<\min \left\{\frac{g_{0}}{4}, \frac{l g_{0}}{16 C_{1}}\right\}
$$

Thus, we have

$$
\frac{g_{0}}{8 C_{1}}<\frac{g_{0}-2 \delta_{2}}{2 C_{1}}
$$

Step 2: As long as $\delta_{2}$ is fixed, we select $\varepsilon_{2}$ such that

$$
0<\varepsilon_{2}<\frac{\delta_{2}}{2 C_{7}}
$$

Then we get

$$
\frac{1}{2}-\frac{\varepsilon_{2} C_{7}}{\delta_{2}}>0
$$

Step 3: Next, we choose $\varepsilon_{1}$ satisfies the relation

$$
\varepsilon_{2} \cdot \frac{g_{0}}{8 C_{1}}<\varepsilon_{1}<\varepsilon_{2} \cdot \frac{g_{0}-2 \delta_{2}}{2 C_{1}}
$$

By (4.23) and (4.25), we get

$$
\varepsilon_{2}\left(g_{0}-\delta_{2}\right)-\varepsilon_{1} C_{1}>\varepsilon_{1} C_{1}+\varepsilon_{2} \delta_{2}>0
$$

and

$$
\varepsilon_{2} \delta_{2}-\frac{\varepsilon_{1} l}{2}<0
$$

Step 4: Now, we must ensure that the first inequality and the fourth one in (4.22) hold. That is to say, for the positive constants $|\mu|, \sigma, \xi$, the following system of inequalities

$$
\left\{\begin{array}{l}
\frac{1}{2}|\mu|+\frac{1}{2} \xi<\varepsilon_{2}\left(g_{0}-\delta_{2}\right)-\varepsilon_{1} C_{1}, \\
-\frac{1}{2}|\mu|+\frac{1}{2 e^{\sigma \tau}} \xi>\varepsilon_{1} C_{1}+\varepsilon_{2} \delta_{2},
\end{array}\right.
$$

must be solvable.

Let $k_{1}=\varepsilon_{2}\left(g_{0}-\delta_{2}\right)-\varepsilon_{1} C_{1}$ and $k_{2}=\varepsilon_{1} C_{1}+\varepsilon_{2} \delta_{2}$. Thus, by (4.26), $k_{1}$ and $k_{2}$ are two positive constants depending on $g_{0}$. And then the system (4.28) becomes

$$
\left\{\begin{array}{l}
|\mu|+\xi<2 k_{1} \\
-|\mu|+\frac{1}{e^{\sigma \tau}} \xi>2 k_{2}
\end{array}\right.
$$

By (4.26), we have $k_{1}>k_{2}$. Note that $e^{\sigma \tau} \rightarrow 1$ as $\sigma \rightarrow 0$. Thus, if we choose $\sigma$ small enough, there exists a positive constant $\xi$ such that

$$
2 k_{2} e^{\sigma \tau}<\xi<2 k_{1}
$$


And then, we have $2 k_{1}-\xi>0$ and $\frac{\xi}{e^{\sigma \tau}}-2 k_{2}>0$.

Thus, the system of inequalities (4.29) is solvable if we choose

$$
|\mu|<\min \left\{2 k_{1}-\xi, \frac{\xi}{e^{\sigma \tau}}-2 k_{2}\right\}=: a .
$$

Here, $a$ is only dependant on $g_{0}$.

Therefore, (4.22) is solvable under the condition (4.31).

Consequently, from (4.21), there exist two positive constants $\gamma_{1}$ and $\gamma_{2}$ such that

$$
\frac{d L(t)}{d t} \leq-\gamma_{1} E(t)+\gamma_{2}(g \circ \nabla u)(t), \quad \forall t \geq t_{0} .
$$

Similar to the steps in [13], the remaining part of the proof of inequality (4.4) can be finished. For reader's convenience, we here write the details as follows:

By multiplying (4.32) by $\zeta$, we arrive at

$$
\zeta \frac{d L(t)}{d t} \leq-\gamma_{1} \zeta E(t)+\gamma_{2} \zeta(g \circ \nabla u)(t), \quad \forall t \geq t_{0} .
$$

Recalling (A2), (4.2), (4.11) and the first inequality in (4.29), we get

$$
\begin{aligned}
\zeta \frac{d L(t)}{d t} & \leq-\gamma_{1} \zeta E(t)-\gamma_{2}\left(g^{\prime} \circ \nabla u\right)(t) \\
& \leq-\gamma_{1} \zeta E(t)-2 \gamma_{2} E^{\prime}(t)+2 k_{1} \gamma_{2}\left\|u_{t}\right\|_{2}^{2} \\
& \leq-\gamma_{1} \zeta E(t)-2 \gamma_{2} E^{\prime}(t)+4 k_{1} \gamma_{2} E(t), \quad \forall t \geq t_{0} .
\end{aligned}
$$

Now, we add a restriction condition on $\zeta$, that is, we suppose that

$$
\zeta>\frac{4 k_{1} \gamma_{2}}{\gamma_{1}}
$$

Then, there exists a positive constant $\gamma_{3}$ such that

$$
F^{\prime}(t) \leq-\gamma_{3} \zeta E(t)
$$

where

$$
F(t)=\zeta L(t)+2 \gamma_{2} E(t) \sim E(t) .
$$

And then, there exists a positive constant $\gamma_{4}$ such that

$$
F^{\prime}(t) \leq-\gamma_{3} \zeta E(t) \leq-\gamma_{4} \zeta F(t)
$$

A simple integration of $(4.38)$ over $\left(t_{0}, t\right)$ leads to

$$
F(t) \leq F(0) e^{-\gamma_{4} \zeta\left(t-t_{0}\right)}, \quad \forall t \geq t_{0} .
$$

A combination of (4.37) and (4.39) leads to (4.4). The proof of Theorem 4.1 is thus completed. $\square$

Remark 4.3 We here point out that the restriction condition (4.35) only makes the admissible space of the function $g$ a little narrow. But it is not difficult to find that the constant $\zeta$ can be extended to the case $\zeta=\zeta(t)$, only if $\zeta(t)$ is a positive nonincreasing differentiable function with lower bound. 


\section{Open problem}

What results can we hope to get in the case $0<\mu_{1}<\mu_{2}$ ? We first recall that, in the absence of time-delay, the energy of the problem (1.2) is dissipative. That is to say, the damping item is a "good" item for the energy decay. So it is not difficult to imagine that, if $\left|\mu_{1}\right|<\mu_{2}<a$ (Here, $a$ is defined by (4.31)), we also can obtain the energy decay result. But, if $a<\left|\mu_{1}\right|<\mu_{2}$, it could be instability. And this problem is still open.

\section{Acknowledgments}

The authors would like to express their sincere gratitude to the anonymous referee for his/her valuable comments and useful suggestions on the manuscript of this work.

\section{References}

[1] J. Hale and S. Verduyn Lunel, "Introduction to Functional Differential Equations", Volume 99 of "Applied Mathematical Sciences", Springer-Verlag, New York., 1993.

[2] R. Datko, J. Lagnese, M.P. Polis, An example on the effect of time delays in boundary feedback stabilization of wave equations, SIAM J.Control Optim, 1986, 24:152-156.

[3] R. Datko, Not all feedback stabilized hyperbolic systems are robust with respect to small time delays in their feedbacks, SIAM J.Control Optim, 1988, 26:687-713.

[4] R. Datko, Two examples of ill-posedness with respect to time delays revisited methods, IEEE Trans. Automat. Control, 1997,42:511-515.

[5] G.Q. Xu, S.P. Yung and L.K. Li, Stabilization of wave systems with input delay in the boundary control, ESAIM Control Optim. Calc.Var., 2006,12(4):770-785.

[6] S. Nicaise, C. Pignotti, Stability and instability results of the wave equations with a delay term in the boundary or internal feedbacks, SIAM J.Control Optim, 2006, 45:1561-1585(electronic).

[7] S. Nicaise, J. Valein, Stabilization of the wave equation on 1-D networks with a delay term in the nodal feedbacks, Netw. Heterog. Media, 2007,2(3):425-479(electronic).

[8] S. Nicaise, C. Pignotti, Stabilization of the wave equation with boundary or internal distributed delay, Diff. Int. Equs., 2008,21(9-10):935-958.

[9] M.M. Cavalcanti, V.N. Domingos Cavalcanti, J.A. Soriano, Exponential decay for the solution of semilinear viscoelastic wave equations with localized damping, E. J. Differ. Int. Equ., 2002,44:1-14.

[10] S. Berrimi, S.A. Messaoudi, Existence and decay of solutions of a viscoelastic equation with a nonlinear source, Nonl. Anal., 2006, 64:2314-2331.

[11] F. Alaban-Boussouira, P. Cannarsa, D. Sforza, Decay estimates for second order evolution equations with memory. J. Funct. Anal., 2008,254(5):1342-1372.

[12] M.M. Cavalcanti, H.P. Oquendo, Frictional versus viscoelastic damping in a semilinear wave equation, SIAM J.Control Optim., 2003,42(4):1310-1324.

[13] M. Kirane, B. Said-Houari, Existence and asymptotic stability of a viscoelastic wave equation with a delay, Z. Angew. Math.Phys., 2011,62:1065-1082.

[14] W.J. Liu, General decay of the solution for viscoelastic wave equation with a time-varying delay term in the internal feedback, arXiv: 1208.4007v1, 20 Aug, 2012.

[15] J.E. Munoz Rivera, M. Naso, E. Vuk, Asymptotic behavior of the energy for electromagnetic system with memory, J. Math. Meth. Appl. Sci., 2004, 25(7):819-941. 
[16] S.A. Messaoudi, General decay of solutions of a viscoelastic equation, J. Math. Anal. Appl., 2008, 341:1457-1467.

[17] J.-L. Lions, Quelques méthodes de résolution des problèmes aux limites non linéaires. Dunod, 1969.

[18] N.-e. Tatar, Arbitrary decay in linear viscoelasticity, J. Math. Phys., 2011, 52: 01350201-01350212.

[19] M.I. Mustafa, S.A. Messaoudi, General stability result for viscoelastic wave equations, J. Math. Phys., 2012, 53: 05370201-05370214.

[20] M. Gugat, Boundary feedback stabilization by time delay for one-dimensional wave equations, IMA $J$ Math Control Info, 2010, 27(2):189-203.

[21] M. Gugat, G. Leugering, Feedback stabilization of quasilinear hyperbolic systems with varying delays, Methods and Models in Automation and Robotics(MMAR), 2012 17th International Conference on, 27-30 Aug. 2012:125-130.

[22] C. Pignotti, A note on stabilization of locally damped wave equations with time delay, Systems \& Control Letters, 2012, 61(1):92-97.

[23] L. C. Evans, Partial Differential Equations, Graduate Studies in Mathematics, Volume 19,American Mathematical Society, 1997. 